\title{
Hiperplasia coronoidea bilateral. Una rara patología causante de disfunción temporomandibular
}

\section{Bilateral coronoid hyperplasia. An uncommon pathology cause of temporomandibular disorders}

\author{
Eguia del Valle A*, Uribarri Etxebarria A, Martínez-Conde Llamosas R, \\ López Vicente J, Ginestal E**, Aguirre Urizar JM*
}

\section{RESUMEN}

La hiperplasia coronoidea es una condición patológica muy poco frecuente, caracterizada por un crecimiento exagerado de la apófisis coronoides mandibular unilateral o bilateral. Desde la primera descripción realizada por Langenbeck a mediados del siglo XIX han aparecido descritos ocasionalmente en la literatura nuevos casos de esta rara alteración. Aunque en ocasiones es asintomática, su principal manifestación clínica es la restricción de la movilidad mandibular, principalmente en los movimientos de apertura y protrusión. Dicha limitación se debe a la impactación de la apófisis coronoides con el hueso cigomático.

En este trabajo se presenta el caso clínico de un varón de 30 años de edad sin antecedentes médicos de interés que acude a consulta por un cuadro de limitación de apertura progresiva y dolor en la región temporomandibular. Tras la exploración, el empleo de técnicas radiológicas convencionales y especiales permitieron confirmar el diagnóstico de hiperplasia coronoidea bilateral. Así mismo, en este trabajo se recogen los principales aspectos clinicopatológicos y diagnósticos de esta alteración. Se realiza un repaso de los aspectos más importantes en el diagnóstico diferencial de los procesos que cursan con limitación de la apertura bucal. Dentro de este diagnóstico diferencial, y a pesar de su baja prevalencia, debe incluirse esta patología.

Palabras clave: Hiperplasia coronoidea, limitación apertura, articulación temporomandibular, disfunción temporomandibular.

\section{SUMMARY}

Coronoid hyperplasia is an uncommon pathology characterized by an excessive uni or bilateral growth of the mandibular coronoid process. Since the first description by Langenbeck in the middle of the XIX century, many cases have been reported in the literature. In some cases patients are asymptomatic. Nevertheless, in most of them a limitation in the mouth movements can be observed, especially during the opening and protrusion movements. This limitation is caused by the impact of the coronoid process with the zigomatic bone.

In this paper we present the case of a 30 years old male patient, without other medical history, that was remitted to our service because of the presence of severe pain in the temporomandibular area and a progressive limitation of the mouth opening. After the clinical exploration a radiographic exam was carried out. Based on them the patient was diagnosed of bilateral coronoid hyperplasia. We also review the most important aspects on this disease, especially those related to the differential diagnosis with other pathologies causing limitation of mouth movements. Coronoid hyperplasia must be always considered in the differential diagnosis in cases of progressive mouth movement limitation, although this is a really uncommon pathology.

* Medicina Bucal. Facultad de Medicina y Odontología. Universidad del País Vasco / EHU. Leioa (Vizcaya). España.

** Profesor titular. Facultad de Medicina y Odontología. Universidad del País Vasco / EHU. Leioa (Vizcaya). 
Key words: Coronoid hyperplasia, opening limitation, temporomandibular joint, temporomandibular disorders.

Fecha de recepción: 1 de marzo de 2010.

Aceptado para publicación: 22 de marzo de 2010.

Eguia del Valle A, Uribarri Etxebarria A, Martínez-Conde Llamosas R, López Vicente J, Ginestal E, Aguirre Urizar JM. Hiperplasia coronoidea bilateral. Una rara patología causante de disfunción temporomandibular. $A v$. Odontoestomatol 2011; 27 (5): 241-244.

\section{INTRODUCCIÓN}

La hiperplasia coronoidea es una condición patológica muy poco común, caracterizada por un crecimiento desproporcionado y progresivo, tanto en volumen como en altura, de la apófisis coronoides mandibular unilateral o bilateral (1-3). Aunque en ocasiones es asintomática, su principal manifestación clínica es la restricción de la movilidad mandibular, principalmente en los movimientos de apertura y protrusión. Dicha limitación se debe a la impactación de la apófisis coronoides contra el hueso cigomático (1-3). En este trabajo se presenta un nuevo caso clínico de esta infrecuente patología.

\section{CASO CLÍNICO}

Varón de 30 años de edad sin antecedentes médicos de interés que acude a consulta por un cuadro de limitación de apertura progresiva y dolor en la región temporomandibular. El paciente refiere que siempre ha tenido un cierto grado de limitación, pero que este ha ido acrecentándose en los últimos años. No refiere traumatismos en el territorio facial, ni puede relacionar el comienzo del cuadro ni su progresión con ningún acontecimiento.

En la exploración se observa una limitación de la apertura bucal severa, con una apertura máxima de 19 mm sin laterodesviación y end-feel fijo o duro (Figura 1).

Se solicita una ortopantomografía (Figura 2) en la que se observa una elongación manifiesta de ambas

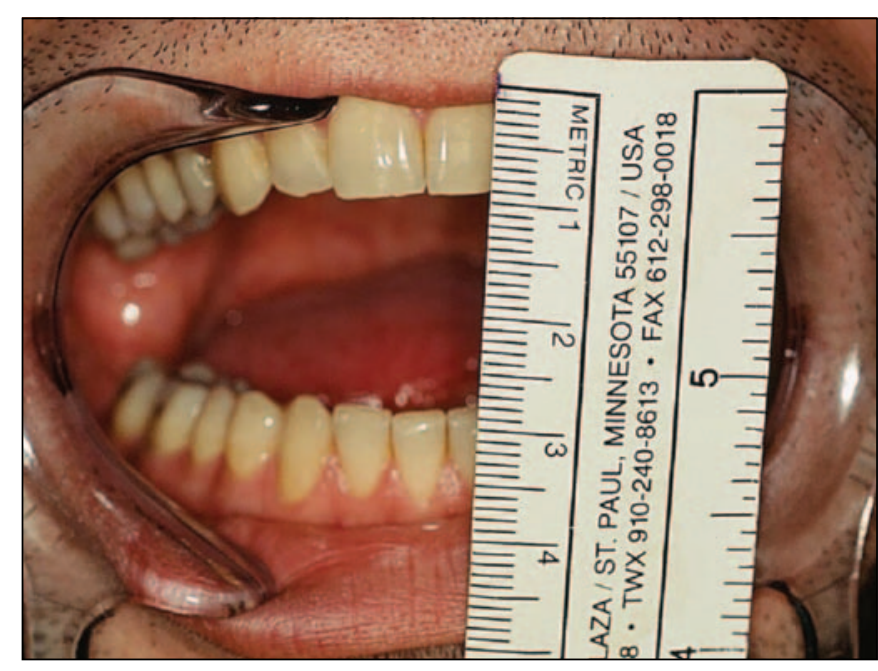

Fig. 1. Limitación apertura bucal $<20$ mm.

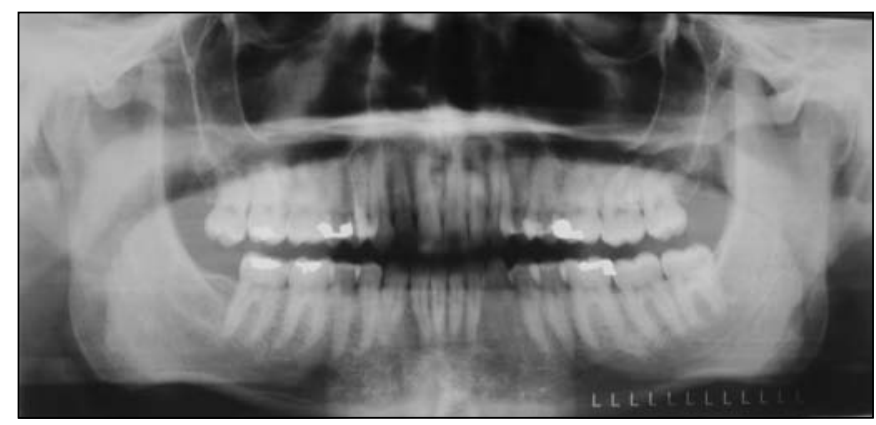

Fig. 2. Ortopantomografía.

apófisis coronoides. Tras ello se solicita una resonancia magnética de ambas ATM para descartar la otras alteraciones (Figura 3) y una tomografía computarizada en la que se puede valorar mejor el tamaño de las apófisis coronoides (Figura 4). 


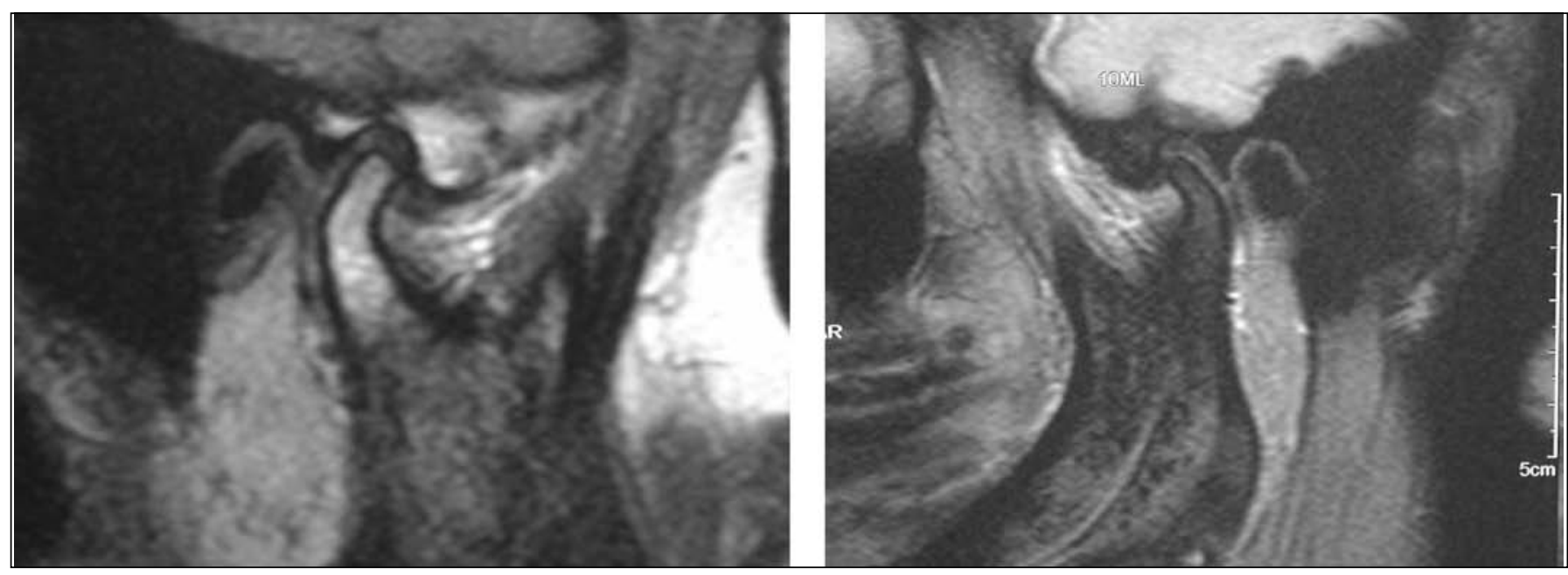

Fig. 3. RM de la ATM izda. y dcha. (boca cerrada).

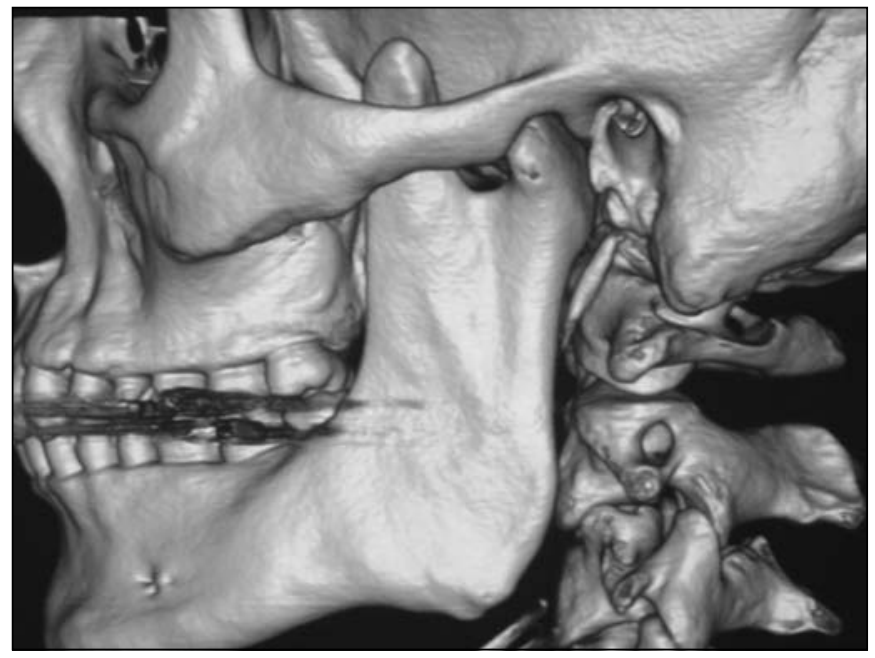

Fig. 4. Reconstrucciones 3D de la TC del paciente. ATM dcha. en boca cerrada.

En base a los resultados de las exploraciones complementarias se establece el diagnóstico de hiperplasia coronoidea bilateral. Se propone al paciente la realización de una coronoidectomía bilateral, aunque el paciente rehusa el tratamiento quirúrgico por el momento.

\section{DISCUSIÓN}

La hiperplasia coronoidea es una condición patológica muy poco frecuente, con algo más de un centenar de casos registrados en la literatura científica, desde las primeras descripciones clínicas de Langenbeck (1853) y Jacob (1989) hasta la fecha (1-5).

Se produce por un alargamiento o crecimiento exagerado, tanto en volumen como en altura, de las apófisis coronoides, cuyo signo clínico principal es la disminución franca de la apertura bucal, debido a su impactación contra el hueso cigomático. En ocasiones puede acompañarse de dolor y de alteraciones de la ATM (1-5).

Puede presentarse de forma ser uni o bilateral, siendo mucho menos frecuente su forma unilateral (1:4) y mostrando cierta predilección por el lado izquierdo. En los casos unilaterales, puede observarse en ocasiones asimetría facial (1-5).

Es más frecuente en varones que en mujeres y es diagnosticado en la mayoría de casos en la segunda o tercera décadas de la vida, aunque existen casos diagnosticados en edad pediátrica $(6,7)$.

Su etiología es desconocida. La hiperactividad del músculo temporal ha sido descrita por diferentes autores como un factor etiológico relevante, sin embargo este aspecto es controvertido y no aceptado unánimemente (3-5). No presenta características histológicas específicas y el estudio histológico de la apófisis coronoides hiperplásicas sólo muestra tejido óseo normal (1-3). 
El diagnóstico definitivo se obtiene mediante un estudio radiológico completo de la región (8). El diagnóstico diferencial debe realizarse con los procesos que cursan con limitación de la apertura bucal. Las causas más habituales de esta limitación tienen su origen en la articulación temporomandibular y entre ellas se encuentran la luxación discocondilar, la fractura condilar, las alteraciones del crecimiento, los procesos inflamatorios severos, las artrosis, adherencias y anquilosis e incluso algunas neoplasias. Las causas extra-articulares de limitación de apertura bucal son raras en proporción a las anteriores y entre ellas debe incluirse también la Enfermedad de Jacob, cuyos síntomas son similares y en la que además de la hiperplasia coronoidea, se produce una formación articular sinovial entre la apófisis coronoides y el hueso cigomático homolateral $(9,10)$.

El tratamiento habitual consiste en la coronoidectomía, complementada con fisioterapia. Siempre que sea posible y que los condicionantes anatómicos lo permitan, la vía intraoral es la recomendada para realizar coronoidectomía, ya que evita la presencia de cicatrices externas y disminuye el riesgo de lesión del nervio facial. La combinación de tratamiento quirúrgico y fisioterapia ofrece resultados clínicos satisfactorios y son pocas las complicaciones. El empleo de dispositivos como el Therabite han demostrado utilidad en la recuperación de la apertura durante el postoperatorio (11). En nuestro caso el paciente rehusó el tratamiento por el momento, por lo que a la espera de la evolución y tras ser informado de todos los aspectos de su patología se mantendrá en control y revisión periódica.

\section{BIBLIOGRAFÍA}

1. Fernández Ferro M, Fernández Sanromán J, Sandoval Gutierrez J, Costas López A, López de Sánchez A, Etayo Pérez A. Treatment of bilateral hyperplasia of the coronoid process of the mandible. Presentation of a case and review of the literature. Med Oral Patol Oral Cir Bucal. 2008 Sep 1;13(9):595-8.

2. Kursoglu P, Capa N. Elongated mandibular coronoid process as a cause of mandibular hypomobility. Cranio 2006 Jul;24(3):213-6.
3. Tieghi R, Galiè M, Piersanti L, Clauser L. Bilateral hyperplasia of the coronoid processes: clinical report. J Craniofac Surg. 2005 Jul; 16(4):723-6.

4. Satoh K, Ohno S, Aizawa T, Imamura M, Mizutani $\mathrm{H}$. Bilateral coronoid hyperplasia in an adolescent: report of a case and review of the literature. J Oral Maxillofac Surg. 2006 Feb;64(2):334-8.

5. Gross M, Gavish A, Calderon S, Gazit E. The coronoid process as a cause of mandibular hypomobility-case reports. J Oral Rehabil 1997 Oct;24(10):776-81.

6. Jaskolka MS, Eppley BL, van Aalst JA. Mandibular coronoid hyperplasia in pediatric patients. Craniofac Surg 2007 Jul;18(4):849-54.

7. Mano T, Ueyama Y, Koyama T, Nishiyama A, Matsumura T. Trismus due to bilateral coronoid hyperplasia in a child: case report. J Oral Maxillofac Surg 2005 Mar;63(3):399-401.

8. Loh HS, Ling SY, Lian CB, Shanmuhasuntharam P. Bilateral coronoid hyperplasia -a report with a view on its management. J Oral Rehabil 1997 Oct; 24(10):782-7.

9. Capote A, Rodríguez FJ, Blasco A, Muñoz MF. Jacob's disease associated with temporomandibular joint dysfunction: a case report. Med Oral Patol Oral Cir Bucal 2005 May-Jul;10(3):210-4.

10. Zhong SC, Xu ZJ, Zhang ZG, Zheng YH, Li TX, Su K. Bilateral coronoid hyperplasia (Jacob disease on right and elongation on left): report of a case and literature review. Oral Surg Oral Med Oral Pathol Oral Radiol Endod 2009 Mar;107(3):64-7.

11. Gibbons AJ, Abulhoul S. Use of a Therabite appliance in the management of bilateral mandibular coronoid hyperplasia. Br J Oral Maxillofac Surg 2007 Sep;45(6):505-6.

\section{CORRESPONDENCIA}

A. Eguia del Valle

Barrio Sarriena, s/n

48940 Leioa (Vizcaya)

e-mail: aeguiadelvalle@yahoo.es 